\title{
Electromagnetic DM technology meets future AO demands
}

\author{
Roger Hamelinck ${ }^{1, a}$, Nick Rosielle ${ }^{2}$, Maarten Steinbuch ${ }^{2}$, and Niek Doelman ${ }^{1}$ \\ 1 TNO Science and Industry, Stieltjesweg 1, P.O.Box 155, 2600 AD Delft, The Netherlands \\ 2 Technische Universiteit Eindhoven, Den Dolech 2, 5600 MB Eindhoven, The Netherlands
}

\begin{abstract}
New deformable mirror technology is developed by the Technische Universiteit Eindhoven, Delft University of Technology and TNO Science and Industry. Several prototype adaptive deformable mirrors are realized mirrors, up to 427 actuators and $\varnothing 150 \mathrm{~mm}$ diameter, with characteristics suitable for future AO systems. The prototypes consist of a $100 \mu \mathrm{m}$ thick, continuous facesheet on which low voltage, electromagnetic, push-pull actuators impose out-of-plane displacements. The variable reluctance actuators with $\pm 10 \mu \mathrm{m}$ stroke and nanometer resolution are located in a standard actuator module. Each module with 61 actuators connects to a single PCB with dedicated, 16 bit, PWM based, drivers. A LVDS multi-drop cable connects up to 32 actuator modules. With the actuator module, accompanying PCB and multi-drop system the deformable mirror technology is made modular in its mechanics and electronics. An Ethernet-LVDS bridge enables any commercial PC to control the mirror using the UDP standard. Latest results of the deformable mirror technology development are presented.
\end{abstract}

\section{Introduction}

Eindhoven University of Technology, Delft University of Technology and TNO Science and Industry have developed a modular adaptive mirror technology based on electromagnetic actuators. Prototypes have a thin continuous facesheet on which push-pull actuators impose out-of-plane displacements. To keep actuator forces and heat dissipation low, a thin facesheet is chosen as the correction element for its low moving mass and low out-of-plane stiffness. The low voltage, electromagnetic actuators are of the variable reluctance type and are located in an actuator plate. Each actuator has $\pm 10 \mu \mathrm{m}$ stroke and nanometer resolution. Design, realization and testing of prototypes is described in detail in [1].

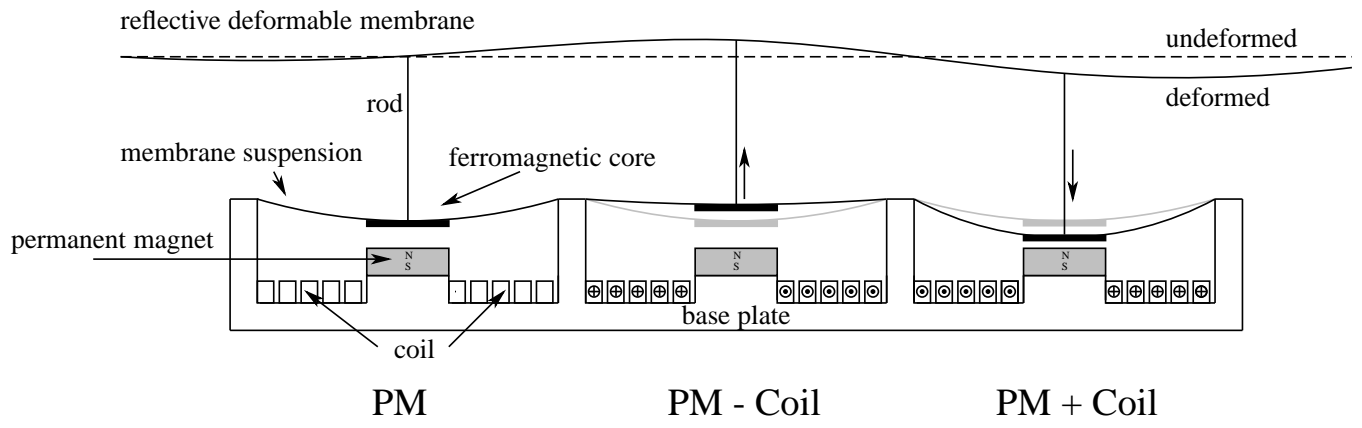

Fig. 1. Schematic of the electromagnetic push-pull actuators and its connection to the mirror facesheet.

\footnotetext{
a e-mail: r.hamelinck@tno.nl
} 


\section{Low voltage, electromagnetic actuators}

Figure 1 shows the schematic of the actuators and the connection to the mirror facesheet. The variable reluctance actuators consist of a closed magnetic circuit in which a permanent magnet (PM) provides static magnetic force on a ferromagnetic core in a membrane suspension. The force is influenced by a current through the coil which is situated around the PM to provide movement of the core. Thin rods are glued between the actuators and the reflective mirror facesheet and link their positions. The bending stiffness of the facesheet yields a smooth reflective surface.

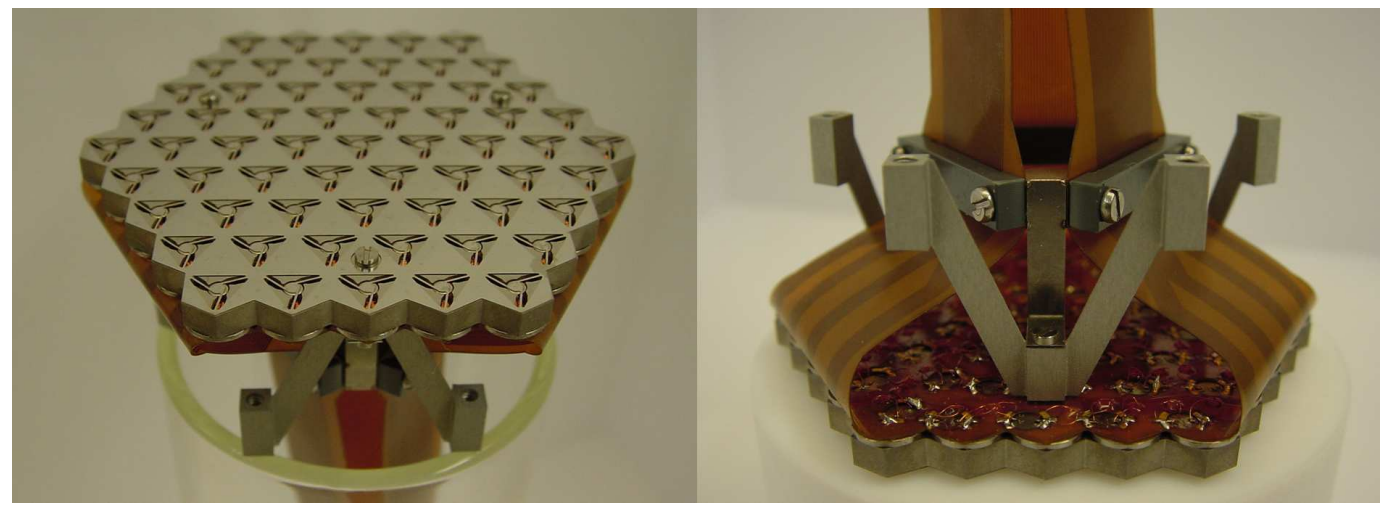

Fig. 2. The standard actuator module with 61 actuators.

\section{A modular system}

The design of a single actuator is translated into a standard module with 61 actuators. This module is shown in Figure 2. In the module a baseplate serves as the flux carrier for the magnetic circuits. Its serrated circumference is made such that the modules can be placed adjacent with a small gap. In the backside of the baseplate holes provide space for inserts, which complete the magnetic circuit
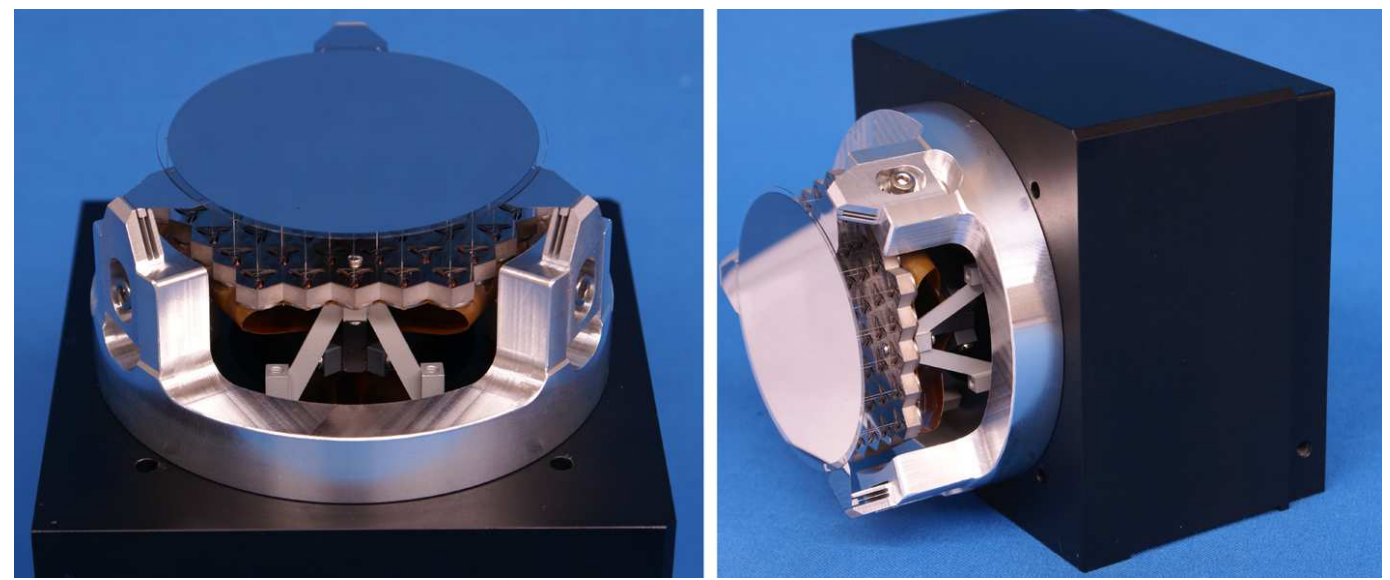

Fig. 3. The $\varnothing 50 \mathrm{~mm}$ adaptive deformable mirror. The $0.1 \mathrm{~mm}$ Pyrex facesheet is deformed by the 61 actuators from a standard actuator module as shown in Figure 2. Three folded leafsprings constrain the in-plane DOF of the mirror facesheet. 

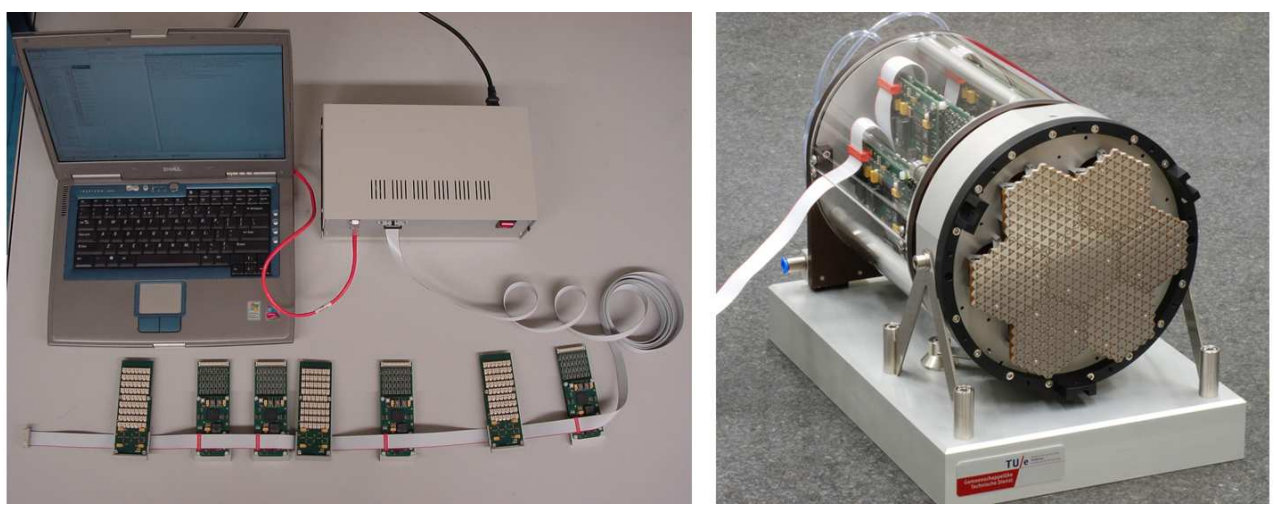

Fig. 4. Left: the 40Mbit/s multi-drop LVDS system. The control PC is connected via the Ethernet-LVDS bridge to dedicated PCBs. Each PCB holds 61, 16 bits, PWM based drivers. Up to 32 PCBs can be connected. Right: the modularity shown in the actuator modules: 7 actuator modules are placed adjacent to form a 427 actuator grid.

on each actuator. The coils, made from $50 \mu \mathrm{m}$ copper wire, with 500 turns, are glued on the inserts together with the permanent magnets. A flex foil is designed to connect the coil wires to the driver PCB. A strain relief prevents the soldered connections from damage. The actuator grid is connected with three A-frames to its support structure. Figure 3 shows a $\varnothing 50 \mathrm{~mm}$ mirror connected with a single actuator module. The dedicated driver PCB holds 61, 16 bits, PWM based drivers. Up to 32 PCBs can be connected to a single multi-drop cable (Figure 4). To minimize power consumption at the receiving ends and maximize design freedom, the multi-drop low-voltage differential signaling (LVDS) connection is chosen. This full duplex line operates at 40Mbit/s. Modularity of the actuators and the accompanying electronics is shown on the right in Figure 4, where 7 modules form the actuator grid with 427 actuators in a $\varnothing 150 \mathrm{~mm}$ mirror prior to assembly of the mirror facesheet. In Figure 6 the fully assembled mirror is shown with its $\varnothing 150 \mathrm{~mm}$ facesheet. With the actuator module and accompanying PCB and multi-drop system the deformable mirror technology is made modular in its mechanics and electronics.

\section{Performance}

With the first mechanical resonance at $\approx 1 \mathrm{kHz}$, the mirrors are suitable for a closed loop control bandwidth of $200 \mathrm{~Hz}$. This resonance does not decrease as the mirror diameter is extended because the stiffness per unit area is constant. Figure 6 shows on the left a single influence function as measured

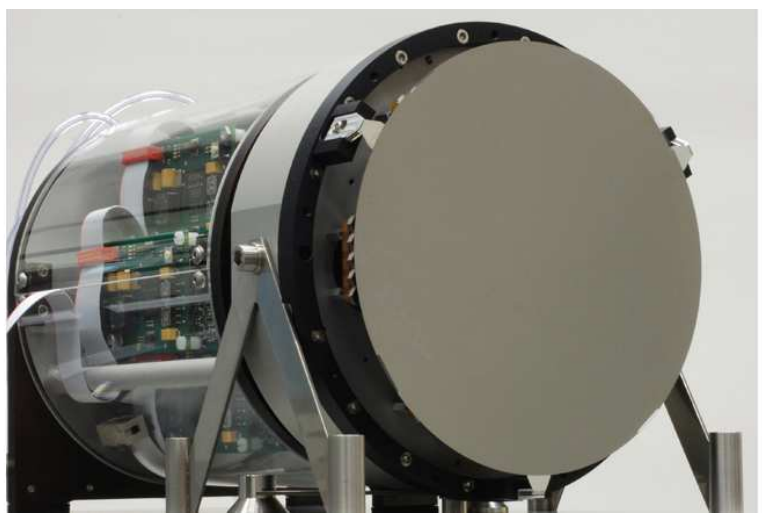

Fig. 5. The assembled $\varnothing 150 \mathrm{~mm}$ deformable mirror with the face sheet connected to the actuator modules. 

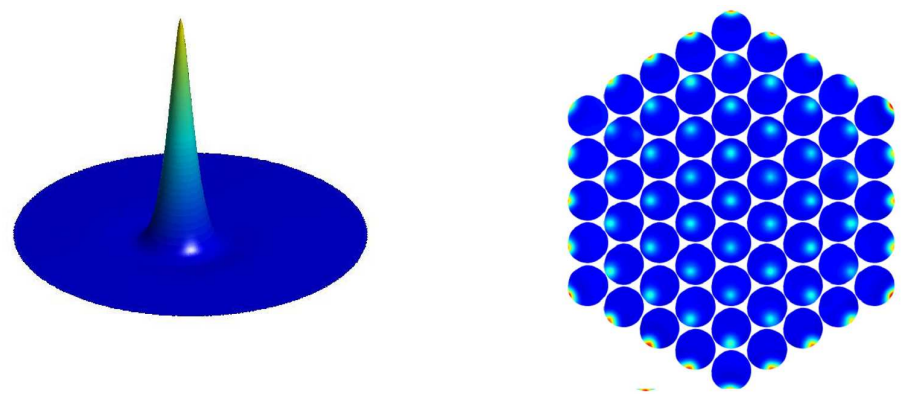

Fig. 6. Left: a measured influence function of one of the central actuators of the mirror shown in Figure 3. Right: 61 measured influence functions. Each influence function is scaled and placed on its specific actuator position.

from one of the central actuators of the mirror shown in Figure 3. On the right in Figure 6 all 61 measured influence functions. Each influence function is scaled and placed on its specific actuator position. As expected there is a 60 degree symmetry and the influence functions at the circumference have a larger amplitude than the inner actuators, because the mirror has a free edge. Figure 7 shows a Zernike table, put together by measurements from the 61 actuator mirror. Each Zernike mode differs $\approx 20 \mathrm{~nm}$ rms from perfect. This is due to the limited number of actuators and the glued actuator mirror connection $(3 \mathrm{~nm} \mathrm{rms})$.

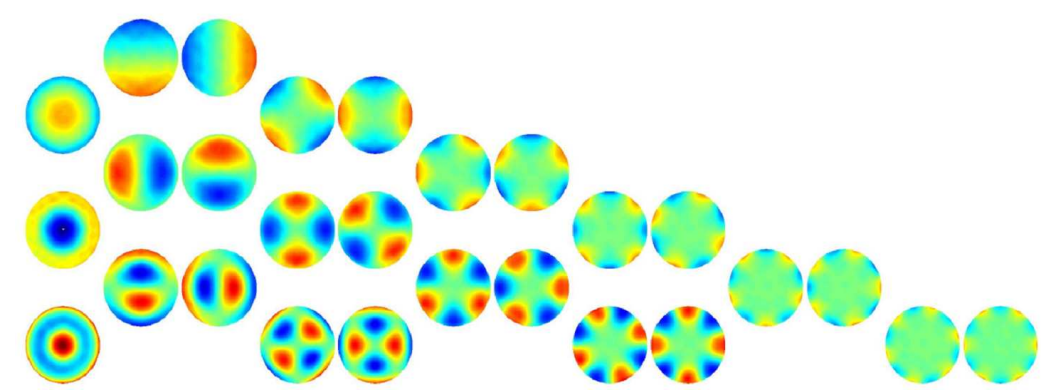

Fig. 7. Zernike modes, measured from a $\varnothing 50 \mathrm{~mm}$ mirror with 61 actuators. The measured modes differ $\approx 20 \mathrm{~nm}$ rms from the perfect Zernike mode.

\section{Conclusion}

DM-technology based on efficient, low voltage, electromagnetic, push-pull actuators is presented. Because of its modularity in mechanics and electronics, it suits future AO deformable mirror requirements, where large numbers of high performance actuators are needed at reasonable costs.

\section{Acknowledgements}

The work is supported by the Dutch Innovative Research Project (IOP) Precision Technology.

\section{References}

1. Hamelinck et al, Validation of a new adaptive deformable mirror concept, Proceedings of SPIE, 2008, (Volume 7015) 